\title{
EXTENDING CLUSTERSIM WITH MP AND DSM MODULES
}

\author{
Christiane Pousa, Luiz E. Ramos, Luís F. Góes, Carlos A. Martins \\ Graduation Program in Electrical Engineering, Pontifical Catholic University of Minas \\ Gerais, Belo Horizonte, Minas Gerais, Brazil
}

\begin{abstract}
In this paper, we present a new version of ClusterSim (Cluster Simulation Tool), in which we included two new modules: Message-Passing (MP) and Distributed Shared Memory (DSM). ClusterSim supports the visual modeling and the simulation of clusters and their workloads for performance analysis. A modeled cluster is composed of single or multi-processed nodes, parallel job schedulers, network topologies, message-passing communications, distributed shared memory and technologies. A modeled workload is represented by users that submit jobs composed of tasks described by probability distributions and their internal structure (CPU, I/O, DSM and MPI instructions). Our main objectives in this paper are: to present a new version of ClusterSim with the inclusion of Message-Passing and Distributed Shared Memory simulation modules; to present the new software architecture and simulation model; to verify the proposal and implementation of MPI collective communication functions using different communication patterns (Message-Passing Module); to verify the proposal and implementation of DSM operations, consistency models and coherence protocols for object sharing (Distributed Shared Memory Module); to analyze ClusterSim v.1.1 by means of two case studies. Our main contributions are the inclusion of the Message-Passing and Distributed Shared Memory simulation modules, a more detailed simulation model of ClusterSim and new features in the graphical environment.
\end{abstract}

Key words: Cluster Computing, Discrete-Event Simulation, Distributed Shared Memory, Message-Passing Communications, Performance Analysis 


\section{INTRODUCTION}

Nowadays, clusters of workstations are widely used in academy, industry and commerce. Usually built with "commodity-off-the-shelf" hardware components and freeware or shareware available in the web, they are a low cost and high performance alternative to supercomputers ${ }^{1,2}$. The performance analysis of different DSM software, message passing libraries, parallel job scheduling algorithms, consistency models, interconnection networks and topologies, heterogeneous nodes and parallel jobs on real clusters is complex. It requires: a long time to develop and change software; a high financial cost to acquire new hardware; a controllable and stable environment; less intrusive performance analysis tools etc. On the other hand, analytical modeling for the performance analysis of clusters requires too many simplifications and assumptions ${ }^{3}$.

Thus, simulation is a less expensive (financial cost) performance analysis technique than measurement on real systems. Besides, simulation is also more accurate than analytical modeling for evaluating the performance of a cluster and constructive blocks. It allows a more detailed, flexible and controlled environment. It makes feasible for researchers to compare a wide variety of clusters configurations and workloads ${ }^{3,4}$. Besides, there are many available types of tools that aid the development of simulations: simulation libraries (SimJava ${ }^{5,6}$, JSDESLib $^{1}$ etc), languages (SIMSCRIPT ${ }^{4}, \mathrm{SLAM}^{4}$ etc) and application specific simulation tools $\left(\right.$ Gridsim $^{3}$, Simgrid $^{7}$, SRGSim $^{8}$, MPI-SIM ${ }^{9}$, PP-MESS-SIM ${ }^{10}$, DSMSim $^{11}$ etc).

In this paper, we present the implementation of the new version of Cluster Simulation Tool (ClusterSim v.1.1) that is used to model clusters and their workloads through a graphical environment, and evaluate their performance using simulation. The tool is an evolution of RJSSim ${ }^{12}$ and ClusterSim v. $1.0^{13}$. This new version was extended with two new modules: the Message-Passing Module (MP module) and the Distributed Shared Memory Module (DSM module). It also presents a more detailed simulation model and some new features in the graphical environment. The main objectives of this paper are: to present and verify both new modules (MP and DSM) of ClusterSim v.1.1.; to analyze ClusterSim by means of two case studies. The main goals of this paper are: the proposal and implementation of two new simulation modules for ClusterSim: MP and DSM; the simulation of MPI collective communication functions using different communication patterns (using MP); the proposal and implementation of DSM operations, consistency models and coherence protocols for object sharing (using DSM); the introduction of new features in the graphical environment; and an automatic workload generation module. 


\section{RELATED WORKS}

In the last years, simulation has been used as a powerful technique for performance analysis of computer systems ${ }^{14,15}$. Researchers usually build simplified specific purpose simulation tools and do not describe them in detail or do not make available the source code and/or binaries. Nevertheless some works regarding the simulation of parallel, network, cluster and grid computing make available their simulation tools and documentation $^{3,7,8,9,10,11,16,17,18}$. A detailed analysis of those works is found in our previous papers ${ }^{1,14}$ and other simulation tools can be found in ${ }^{18,19}$.

In this section, we briefly describe the simulation tools that have the closest relation with the new two modules of ClusterSim: a cluster computing simulation tool $\left(\mathrm{SRGSim}^{8}\right)$, two message passing simulators (MPI-SIM $^{9}$ and PP-MESS-SIM ${ }^{10}$ ) and two DSM simulation tools $\left(\right.$ DSMSim $^{11}$ and $\mathrm{Mica}^{20}$ ), which present interesting features.

SRGSim is a Java-based discrete-event simulation tool developed in the University of California. It simulates some classes of parallel job scheduling (dynamic and static scheduling), architectures (cluster of computers, multiprocessors etc) and jobs through probabilistic models, constants and DAGs (Direct Acyclic Graphs). The main features of SRGSim are: a DAG editor, the description of jobs by means of traces, probabilistic models or DAGs, the use of CPU-, I/O- and Communication-bounded jobs, the support to some network topologies, and its parallel and distributed implementation. SRGSim has a text-only interface and does not support multithreading. Moreover, it does not support heterogeneous nodes and mechanisms that simulate the MPI communication functions ${ }^{8}$.

MPI-SIM $^{9}$ is a library in $\mathrm{C}$ for the execution-driven parallel (multithreaded) simulation of MPI programs. The simulated workload can use a subset of available MPI point-to-point primitives and collective communication functions. MPI-SIM predicts the performance of the MPI programs as a function of architectural characteristics (number of processors, network latencies etc). The simulator supports point-to-point networks only.

PP-MESS-SIM ${ }^{10}$ is a C++ object-oriented event-driven simulator for message passing in multicomputers. Its main features are: the support to different router architectures, the possibility of multiple coexisting routing policies and switching schemes, the extensibility, the representation of traffic classes and workloads as distribution functions and the use of an input language (a text-only interface).

DSMSim $^{11}$ is a C++- distributed shared memory simulation tool developed in the University of Wayne State. In DSMSim a workload can only be represented by real programs of String DSM system. The simulation model is based on events and entities. Only one consistency model and one 
coherence protocol were implemented. DSMSim implements a page-based DSM and decentralized management for shared pages. Besides, the tool does not have a graphical environment for modeling the cluster architecture and uses simple statistical analysis mechanisms.

MICA is a memory and interconnect simulation environment for cachebased architectures. It simulates DSMs that were implemented in hardware and uses real traces as workloads. The simulation is driven by events rather than by system clocks, which in turn speeds up the simulation whereas preserving accuracy. MICA was built on top of $\operatorname{CSIM}^{20}$, which supports the use of a variety of scheduling policies and complex systems. Since CSIM provides user-level threads, it can simulate a set of computing nodes, pointto-point and collective communication activities through these threads. MICA provides: cache, bus, memory, directory, node controller/network interface and interconnection modules. It implements two page allocation policies and some routing algorithms ${ }^{21}$.

In spite of the SRGSim has a detailed probabilistic workload simulation model, its model does not provide a detailed structural description of a job (ex: loop structures, different communications functions etc). Moreover, the workload probabilistic description is less representative, but it requires less simulation time. Thus, the structural description of the workload makes ClusterSim more representative. Besides, its graphical environment makes it easier to use. As well as SRGSim, ClusterSim implements CPU, I/O and communication functions that support the definition of different communications patterns and algorithms models with varying granularity.

ClusterSim simulates MPI collective communication functions with basis on point-to-point primitives like PP-MESS-SIM and MPI-SIM. However, MPI-SIM requires real $\mathrm{C} / \mathrm{C}++/ \mathrm{UC}$ programs in order to run a simulation, whereas ClusterSim uses a hybrid language independent workload model. Besides, unlike ClusterSim and PP-MESS-SIM, MPI-SIM only simulates point-to-point networks. On the other hand, PP-MESS-SIM uses a specific language for workload definition, instead of a graphical environment.

As well as DSMSim and Mica, ClusterSim can simulate distributed shared memory (DSM). Nevertheless, Mica only simulates DSM implemented in hardware, which uses pages as grains (invariable granularity). ClusterSim simulates a variety of consistency models, access protocols, coherence protocols and some synchronization primitives that were not found in DSMSim. Both DSMSim and Mica, just accept real programs as input, unlike ClusterSim in which real and abstract DSM programs can be simulated. 


\section{CLUSTER SIMULATION TOOL (CLUSTERSIM)}

ClusterSim is a Java-based parallel discrete-event simulation tool for cluster computing. It supports the visual modeling and the simulation of clusters and their workloads for performance analysis. A modeled cluster is composed of single or multi-processed nodes, parallel job schedulers, network topologies, distributed shared memory, message-passing mechanisms and technologies. A workload is represented by users that submit jobs composed of tasks described by probability distributions and their internal structure.

The main features of ClusterSim v.1.0 (old version) are: a graphical environment to model clusters and their workloads; an available source code; extensible classes; a mechanism to implement new job scheduling algorithms, network topologies, etc; representation of a job by some probability distributions and internal structure (loop structures, CPU, I/O, MPI (communication) instructions); support to the modeling of clusters and heterogeneous or homogeneous nodes; an independent thread for each simulation entity (architectures and users); the support to some collective and point-to-point MPI (Message Passing Interface) functions; representation of a network by its topology, latency, bandwidth, protocol overhead, error rate and maximum segment size; the support to different parallel job scheduling algorithms (space sharing, gang scheduling, etc) and node scheduling algorithms (first-come-first-served (FCFS), etc); a statistical and performance module that calculates some metrics (mean nodes utilization, mean simulation time, mean jobs response time etc); the support to some probability distributions (Normal, Exponential, Erlang HyperExponential, etc) to represent the parallelism degree of the jobs and the interarrival time between jobs submissions and the possibility of specifying the simulation time and seed.

ClusterSim v.1.0 didn't have distributed shared memory and the message-passing functions were implemented with unique communication patterns. Besides, the user must model the message-passing workloads manually. By including a couple of modules (MP and DSM) the new features of ClusterSim v.1.1 are:

- The support to different communication patterns (ring, binomial tree, etc), memory consistency models (sequential, weak, etc) and coherence protocols (write-invalidate eager, write-update lazy, etc);

- A graphical environment for modeling clusters and workloads that use distributed shared memory;

- A graphical environment for the automatic modeling of message-passing workloads. 


\subsection{The Architecture of ClusterSim}

The architecture of ClusterSim is divided into three layers: graphical environment, entities and core. The first layer allows the modeling and the simulation of clusters and their workloads by means of a graphical environment. Moreover, it provides statistical and performance data about each executed simulation. The second layer is composed of three entities: user, cluster and node. Those entities communicate by means of event exchange supported by the simulation core. The third layer is the simulation core itself, a discrete-event simulation library named JSDESLib ${ }^{1}$.

\subsubsection{Graphical Environment}

The graphical environment was implemented using Java Swing and NetBeans 3.4.1 IDE. ClusterSim v.1.0 is composed of a configuration and simulation execution interface, three workload editors (user, job and task editors) and three architecture editors (cluster, node and processor editors). In the new version of ClusterSim, two new workloads editors (auto job and object editors) were implemented. Besides, some updates were implemented on the existing workload and architecture editors. Using these tools, it is possible to model, execute, save and modify simulation environments and experiments (Fig. 1). As well as ClusterSim editors, the simulation model is divided between workload and architecture.

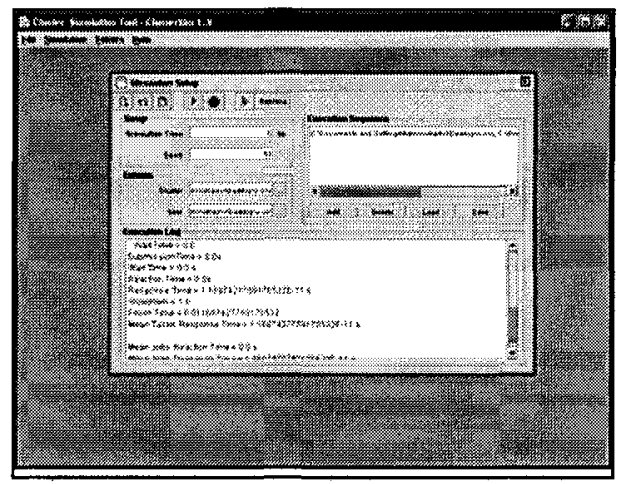

Figure 1. Main interface of the graphical environment

Based on the related works, we chose a hybrid workload model using probability distributions to represent some parameters (parallelism degree and inter-arrival time) and an internal structure description of the jobs. The execution time is a parameter that is commonly found in execution logs, but it is only valid for certain workloads and architectures. Besides, it is 
influenced by many factors such as: load, nodes processing power, network overhead etc. Thus, the execution time of a job must be calculated during a simulation, according to the simulated workload and architecture.

We model a job by describing its internal structure, instead of using its execution time. This approach has many advantages: i) real jobs can be represented directly (without structure modifications); ii) the execution time of a job is dynamically calculated and is only influenced by the simulated environment; iii) the job's behavior is more deterministic; iv) many parallel algorithm models and communication patterns can be implemented.

Unlike execution time, parallelism degree is usually known and indicated by the user that submits a job. As parallelism degree is not influenced by execution time factors, it can be represented by a probability distribution.

In order to avoid long execution traces, the jobs inter-arrival time is also represented by a probability distribution. Moreover, exponential and Erlang hyper-exponential distributions are widely used in the academic community to represent the jobs inter-arrival time ${ }^{14,15}$.

In order to model a workload, we can use the following editors: User, Job or Auto Job, Objects and Task. In the User Editor, it is necessary to specify: the number of jobs to be submitted, the inter-arrival time distribution and the jobs types. The submission probability must be specified for each job type. The sum of those probabilities must be equal to $100 \%$. The jobs types are selected through Monte Carlo's method, where a random number between 0 and 1 is raffled, indicating the job to be submitted. For each new instance of a submitted job type, new values are sampled for the parallelism degree of each task and inter-arrival time.

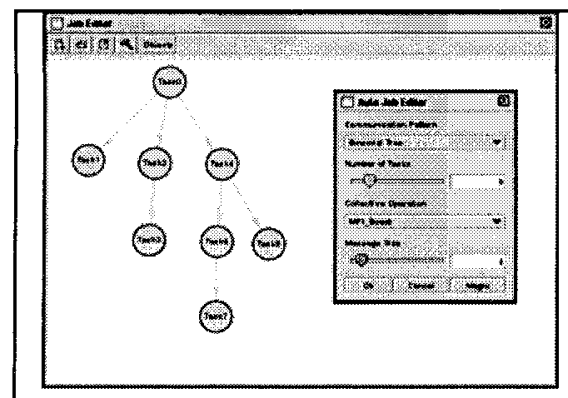

(a)

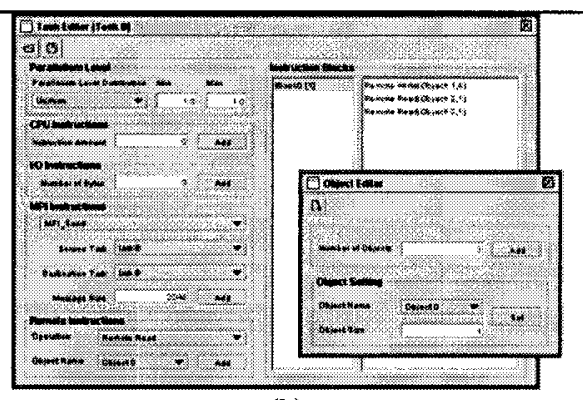

(b)

Figure 2. (a) Job and Auto Job editors; (b) Task and Objects editors

The Job Editor (Fig. 2a) allows the specification of a job through a graph. Each node represents a task and each edge represents the communication between two tasks. Starting from the Job Editor, it is possible to edit each task by activating the Task Editor (Fig. 2b) and create and edit objects for task instructions by activating the Objects Editor. In the Task Editor CPU, 
E/S, DSM and MPI instructions are inserted into instruction blocks and the distribution of the parallelism degree is specified. Each instruction can automatically be parallelized according to the parallelism degree of the task. For instance, suppose a parallel job that follows the process farm model. It would be modeled with two nodes: the master task (parallelism degree equal to 1 ) and the slave tasks (parallelism degree equal to $\mathrm{n}$ ). If the parallelization option was active in the CPU instructions of a slave, the total number of CPU instructions of each slave would be equal to the total number of CPU instructions divided by the parallelism degree $\mathrm{n}$. Thus, it is possible to verify the speedup achieved by the parallelization of a job, without implementing a different job for each parallelism degree.

In order to aid the user on modeling workloads, we implemented an Auto Job Editor (Fig 2a), which automatically creates a job containing a userdefined number of tasks that communicate according to some available communication pattern. We intend to improve that editor in the following versions of ClusterSim.

\subsubsection{Statistical and Performance Module}

For each executed simulation, the statistical and performance module of ClusterSim creates a $\log$ with the calculation of several metrics. The main calculated metrics are: mean jobs and tasks response time; wait, submission, start and end time of each task; number of messages exchanged between nodes (DSM); transmission time (MP); overhead for memory copies; mean jobs slowdown; mean nodes utilization; mean jobs reaction time etc.

\subsubsection{ClusterSim's Entities}

Each entity has specific functions in a simulation environment. The User Entity is responsible for submitting a certain number of jobs to the cluster following a pattern of arrival interval. Besides, each job type has a specific probability of being submitted to the Cluster Entity. This submission is made through the generation of a job arrival event. When the cluster receives this event, it should decide to which nodes the tasks of a job should be directed. So, there is a job management system scheduler that implements certain parallel job scheduling algorithms. When receiving an end of job event, the cluster scheduler should remove the execution job. Fig. 3 shows the events exchange diagram of ClusterSim, detailing the interaction among the User, Cluster and Node entities. To simplify the diagram, some classes were omitted. Other important classes belonging to the cluster entity are: Single System Image, Network, MPI manager and Shared Memory Manager. 


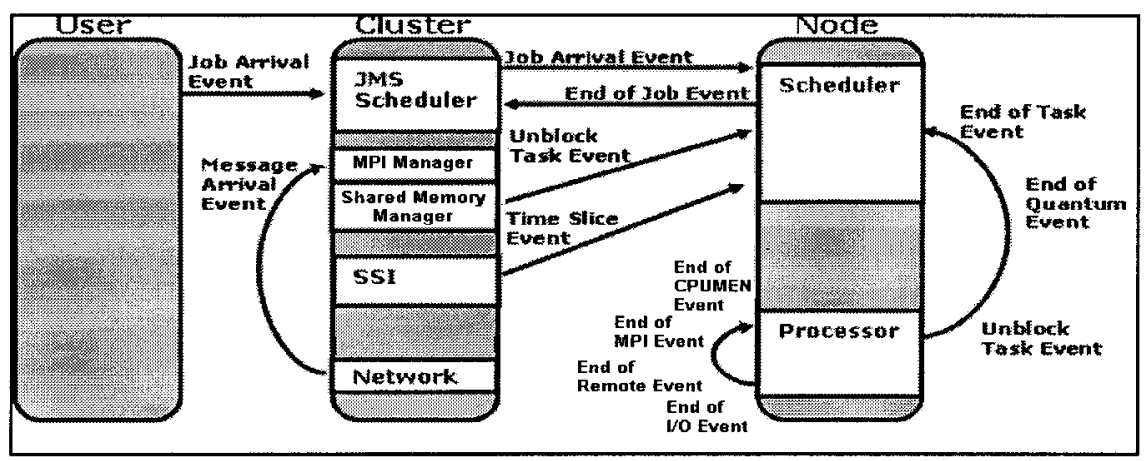

Figure 3. Events exchange diagram of ClusterSim

The Single System Image works as an operating system of the cluster, receiving and directing events to the responsible classes for the event treatment. Besides, it generates periodically the end of time slice event to indicate to the node schedulers that another time slice ended.

The network allows the cluster's nodes to communicate. Network modules include: physical topology, routing policies, message queues, network technology, and performance parameters. The current version offers two types of interconnection topologies: a bus and a switch. Both of them allow only point-to-point communication. The bus provides all nodes with a common medium through which two nodes are able to communicate at a given moment. The other nodes can read the state of the medium and are only allowed to transmit when it is not busy. In this model, no collisions were introduced. Thus, the bus allows only non-simultaneous point-to-point communication. The switch model allows pairs of nodes to communicate simultaneously. However, when a pair of nodes communicates, no other node can transmit or receive from them. Thus, the switch allows up to floor(N/2) simultaneous communications ( $\mathrm{N}$ is the number of cluster nodes).

The network and MPI manager classes implement certain basic communication mechanisms, in order to support message-passing among tasks. As soon as a task executes an MPI function and the MPI manager recognizes it as a sending function, it calls the network class, which generates a message arrival event. The time spent for the message transmission from a node to another depends on the topology, network technology and contention parameters. When a message arrival event reaches its destination, the MPI manager generates a unblock task event, if the task is waiting. Otherwise, the message is stored into a queue.

The Shared Memory Manager classes were implemented for supporting shared memory with DSM write and read operations for objects distributed over a cluster simulated in ClusterSim. As soon as a task executes a DSM operation (Write, Read), the Shared Memory manager interprets the 
operation and executes the correspondent operations micro instructions (SendMulticast, MoveObject, etc). If that micro instruction is a SendMulticast the Shared Memory manager calls the network class, which generates a message arrival event. Like in MPI the time spent for this operation depends on the topology, network technology and contention parameters. When a message arrival event reaches its destiny, the Shared Memory manager generates a unblock task event, if the task is blocked. Otherwise, the message is stored in a queue. After each DSM operation, the Shared Memory manager updates the table of objects at each node.

A cluster entity is composed of several node entities. When the node entity receives a job arrival event from the node scheduler class, it inserts the new job's tasks into a queue. On each clock tick, the scheduler is called and executes the tasks using the processors of the node. An event is generated at the end of each CPU, I/O, DSM or MPI macro instruction within a task. A quantum is assigned to each task. When a task finishes, the processor generates an end of quantum event, so that the node scheduler executes the necessary actions (changing priority, removing the task from the queue etc). When the processor executes all the instructions of a task, an end of task event is generated for the node scheduler.

\subsubsection{ClusterSim's Simulation Core}

The simulation core is composed of the JSDESLib (Java Simple Discrete Event Simulation Library), a Java-based multithreaded discrete-event simulation library, developed by our group. Its main objective is to simplify the development of discrete-event simulation tools ${ }^{1}$.

\subsection{Simulation Model}

A cluster is composed of: homogeneous or heterogeneous nodes, networks, job management systems, single system image, message-passing manager, and distributed shared memory. Each node has a memory hierarchy, I/O devices, one or more processors and an operating system. In our architecture model, a cluster is basically represented by a parallel job scheduler, a network, an MPI Manager, a Shared Memory Manager and a set of nodes.

Overhead $=\left\lceil\frac{\text { MessageSize }}{M S S}\right\rceil \times$ ProtocolOverhead

(Equation 1)

TransmissionTime $=$ Latency $+\frac{(\text { MessageSize }+ \text { Overhead }) \times(1+\text { ErrorRate })}{\text { Bandwidth } \times 10^{6}}$

(Equation 2) 


$$
\text { ElapsedTime }=\text { NumberOfInstructions } \times \text { CyclesPerInstruction } \times \frac{1}{\text { Frequency }}(\text { Equation 3) }
$$

The scheduler implements parallel job scheduling algorithms, such as: space sharing, backfilling, gang scheduling etc. The network is represented by the following parameters: latency (ns), bandwidth $(\mathrm{MB} / \mathrm{s})$, protocol overhead (bytes), error rate (\%), maximum segment size or MSS (bytes) and topology (Fig. 3). The equations 1 and 2 are used for calculating the message transmission time between two nodes. The MPI Manager is represented by MPI point-to-point primitives (blocking and non-blocking) and collective communication functions. And the Shared Memory Manager is represented by several consistency models and coherence protocols.

In our model, each node is represented by the following modules: scheduler, primary and secondary memory transfer rate and processors. The scheduler implements basic scheduling algorithms, such as: Round-Robin and FCFS. It also implements some algorithms that assure the correct operation of the cluster's parallel job scheduling algorithms. The primary memory transfer rate is used by the Shared Memory Manager and MPI manager, when receiving or sending data. The secondary memory transfer rate is used to calculate the time spent in the readings and writings of the $\mathrm{I} / \mathrm{O}$ instructions. A node has one or more processors, and a processor is represented by a clock frequency and cycles per instruction rate (CPI). The processor uses the Equation 3 in order to calculate the elapsed time for executing $n$ instructions of a program.

\section{VERIFICATION OF THE NEW MODULES}

ClusterSim v. 1.0 was verified and validated in ${ }^{1}$ and ${ }^{13}$. In this section, we verify the new simulation modules (MP and DSM), that were constructed on top of already validated modules belonging to ClusterSim v.1.0. In order to verify and test the new modules, we simulated two simple workloads and compared both simulation and analytical results.

The analytical results were obtained using real network values of a cluster (Fast Ethernet, Network Latency: 0.000179s, Data Payload: 1460 bytes, Network Bandwidth: $11.0516 \mathrm{MB} / \mathrm{s}$, Protocol Overhead: 58 bytes, and Primary Memory Transfer Rate: $11.146 \mathrm{MB} / \mathrm{s}$ ) and the equations 1,2 and 3.

The first workload is a set of communication patterns that implement a broadcast operation with point-to-point primitives in a 16 processes job. We simulated the following patterns: Sequential Tree, Binary Tree, Binomial Tree and Chain ${ }^{22,23}$. As they have different parallel fractions, they perform differently over the switch model. Fig. 4 presents the analytical execution diagrams for the referred communication patterns. The diagrams represent 
the communications between processes in time (ms). A pair of cells with the same color in the same column (time interval) means that a pair of processes is communicating within that time interval.

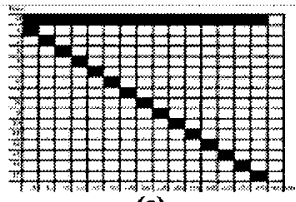

(a)

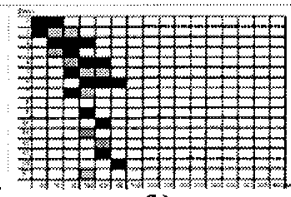

(b)

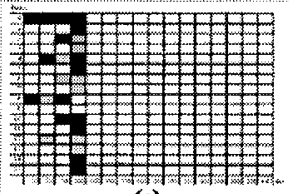

(c)

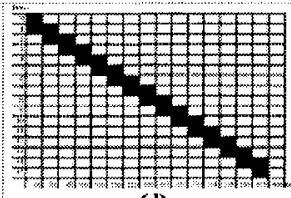

(d)

Figure 4. Analytical execution diagrams for the referred communication patterns: (a) Sequential Tree, (b) Binary Tree, (c) Binomial Tree and (d) Chain

In Fig. 4, we can see that Binomial Tree (c) yields the lowest response time among the analyzed patterns, because it best fits the switch topology of the network. Binary Tree (b) consumes more logical steps than the latter. Sequential Tree does not take advantage of the switch's parallelism because of its sequential nature. The Chain pattern presents the worst results over this network topology because it is sequential and because the dependency between its communications generates extra message transmission overhead.

Table 1. Response time (ms) for a 16 processes job over a switch topology

\begin{tabular}{lccccc}
\hline Comm Pattern & Sequential Tree & Bumaty Tree & Binomial Tree & Chatn \\
\hline Res. Time (ms) & 369.894301 & 147.971813 & 98.656046 & 369.977023 \\
\hline
\end{tabular}

The results obtained in the simulator with 16 processes are presented in Table 1 . They match the analytical results accurately $(0.76 \%$ error), which means that the simulation was successful in this case. We made similar experiments with the reduction and the allgather operations for jobs having different sizes. They matched the analytical execution as well.

The second workload is a set of DSM operations that are managed by strong consistency models and some coherence protocols. First, in order to verify the DSM operations we constructed some jobs containing two tasks that execute DSM operations. Fig. 5 presents the analytical execution diagrams for the referred DSM operations. The diagrams represent the communications between tasks over time (ns). A remote operation is represented by a black line with the name of the operation. The dashed line represents a local DSM operation, which occurs when the node containing the calling task also contains the accessed object. 


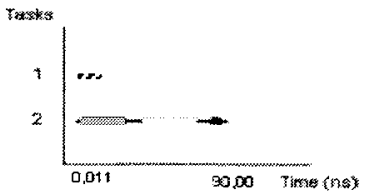

(a)
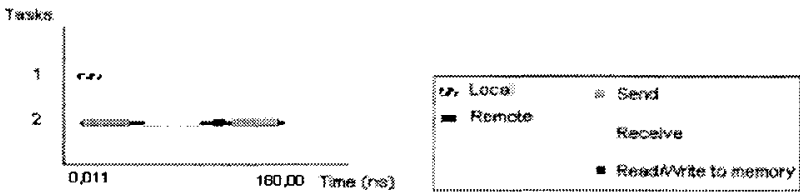

(b)

Figure 5. Analytical execution diagrams: (a) Remote Reads (b) Remote Writes

In Fig. 5, we can see that when a task has the object in its memory the DSM operation becomes local and the response time is lower than when the operation is remote. A remote write operation consumes more time than the local write, because a task has to obtain an object replica, write it, and send the updated object for all other tasks.

Table 2. Response time (ms) for a 2 tasks over a bus topology

\begin{tabular}{ccccc}
\hline Operation & Local Read & Remote Read & Local Write & Remote Write \\
\hline Res. Time $(\mathrm{ms})$ & 0.011687 & 90.49662 & 0.011697 & 180.969828 \\
\hline
\end{tabular}

The results obtained in the simulator with 2 tasks are presented in Table 2. They match the analytical results accurately $(5.8 \%$ error), which means that the simulation was successful in this case.

\begin{tabular}{|c|c|c|c|c|c|}
\hline \multirow{4}{*}{ T1) $\rightarrow$ (T3) } & Fonde & Task & Olhject & Object Yalue & State of Object \\
\hline & $T$ & $T$ & Objecto & o & Velitit \\
\hline & 2 & 2 & Objakt1 & 8 & Valid \\
\hline & 3 & 3 & Object2 & 0 & Volid \\
\hline
\end{tabular}

Figure 6. Job used as the workload in Consistency Model verification

After that verification, we simulated DSM write and read operations on objects, using a sequential consistency model and the following coherence protocols: write-update and write-invalidate. Fig. 6 presents the job that was simulated for the verification of the sequential consistency model and the table that control the shared objects. In this job each task was located in one node and had one object. Each task has tree DSM operations: a Write to its object and two Reads to the objects of the other tasks.

Fig. 6 presents the original location and state of the objects (table of Objects and Nodes) at the beginning of the simulation. Fig. $7 \mathrm{a}$ presents the values in the table of Objects and Nodes after the execution of the Job with the sequential consistency model and the write update coherence protocol. 


\begin{tabular}{|c|c|c|c|c|}
\hline Nade & Takk & Ohiect & Ohject Value & State of Object \\
\hline 7 & $T$ & Objocto, Objoct1, Obyect? & $1,1,1$ & Valie \\
\hline 2 & 2 & Object1, Obj*ct0, Object2 & $1,1,1$ & valia \\
\hline 3 & 3 & Object2, object0, Object! & $1,1,1$ & Valia \\
\hline
\end{tabular}

(a)

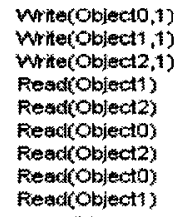

(b)

Figure 7. (a) Table of Objects and Nodes after the job execution (b) Execution Sequence

In ${ }^{24}$, this example was used to show a valid execution sequence for a job executed with sequential consistency model. In that example, a valid execution sequence could be: Write(Object0), Write(Object1), Write(Object2), Read(0), $\operatorname{Read}(2), \operatorname{Read}(1), \operatorname{Read}(2), \operatorname{Read}(0)$ and $\operatorname{Read}(1)$. The resultant objects values for this sequence of execution is: $1,1,1,1,1,1$. As we could see in the Fig. $7 \mathrm{~b}$, our simulation got the same results as the analytical execution presented in ${ }^{24}$.

Thus, we verified the new functions and parts of ClusterSim: MPI collective communication functions (with different communication patterns), DSM operations (write and read), consistency models, coherence protocols. The other functionalities and parts were not verified in this example. The consistency models, MPI collective functions, I/O instructions, network topologies and technologies had been verified exhaustively by means of more specific manual and analytical tests.

\section{EXPERIMENTAL RESULTS}

In this section, we present two case studies. We utilize the MP and DSM modules for cluster computing simulation. Our experimental environment is composed of two 16-node clusters interconnected by Fast Ethernet networks. One of the clusters used a bus interconnection device and the other used a switch. Each node had a Pentium III $1 \mathrm{Ghz}(0.938 \mathrm{GHz})$ processor $(0.9997105 \mathrm{CPI})$. The primary and secondary memories were unlimited and their transfer rates were respectively: $11.146 \mathrm{MB} / \mathrm{s}$ and $23.0 \mathrm{MB} / \mathrm{s}$. The network had a $0.000179 \mathrm{~s}$ latency, a $11.0516 \mathrm{MB} / \mathrm{s}$ bandwidth, a 1460 bytes segment size with a 58 bytes protocol overhead. Those values were obtained from benchmarks and performance libraries (Sandra 2003, PAPI 2.3 etc).

\subsection{Message Passing Operations}

In this case study, we created 10 workloads to test both clusters. The workload is composed of parallel jobs, containing different communication patterns. In order to simplify our experiments, we modeled only three MPI 
collective communication functions using different communication patterns $^{22,23}$. We modeled: MPI_Bcast (one-to-all), MPI_Reduce (all-to-one) and MPI_Allgather (all-to-all). They are often used and are easy to understand and implement. MPI_Bcast and MPI_Reduce were implemented with the following patterns: Sequential, Binary and Binomial trees, and respectively with Chain and Ring. MPI_Allgather was implemented with: Star, FanIn-FanOut, Full FanOut, Circular, Shuffle and Pairwise ${ }^{22,23}$. All patterns were modeled as sets of point-to-point primitives and were used to transmit messages whose sizes were powers-of-two (from 1 Byte to $256 \mathrm{~KB}$ ). We will only present the results for $256 \mathrm{~KB}$ messages, because the other sizes grew proportionally and their analysis would be repetitive.

In the bus based cluster, MPI_Reduce implemented with the Sequential Tree pattern presented the best results (see Table 3a). Ring presented the worst. As this model allows only one transmission at a time, it privileged the patterns with the smallest number of packets (combined messages). As a matter of fact, all patterns sent the same number of packets, but Ring presented an accumulated overhead due to waiting latency. Sequential Tree took advantage of the lack of memory limit. When a packet arrived, the receiving process consumed it as soon as a matching reception primitive was issued. Both Binary and Binomial presented the same results, which are slightly worse than those of Sequential (due to waiting latencies).

In the switch based simulated cluster, Sequential and Ring obtained the same results as in the bus based cluster. As their logics are sequential, they take no advantage of the switch's parallelism. On the other hand, Binomial presented the best results because the number of communicating processes doubled at each step. So this pattern took advantage of the parallelism of the switch, and took less logical steps than Binary.

In the bus based simulated cluster, three patterns presented the best results for MPI_Bcast. As shown in the Table 3b, Sequential, Binary and Binomial trees had the lowest response times for all number of processes and message sizes. They all spent exactly the same time to conclude, because they send N-1 packets and there is no dependency between packet transmissions. On the other hand, Chain presented the worst results due the same effect verified in the Ring reduction.

Over the switch, the dependency problem does not occur, as it does over the bus. So, both the Binary and Binomial trees yielded the same response times as in the reduction operation. The latter presented the best result, because it best explores the parallelism of the switch. Once again, Sequential Tree and Chain presented the same results as they did over the bus.

In the bus based cluster, Circular and Pairwise presented the best result over the bus for MPI_Allgather (see Table 3c). They are logically similar and they transmitted the smallest number of fixed sized packets, among all 
patterns. Shuffle presented a slightly worse performance than the latest couple, because the packet sizes doubled at each step. Besides both bus and switch allow no simultaneous bidirectional exchanges, which requires the steps to be divided in two sequential sub-steps. Over the switch, Pairwise presented the best result, because it takes advantages of parallelism with no bidirectional exchanges. In this case, Shuffle performed better than Circular.

As expected, the results showed that the best pattern for a given situation is the one whose logical topology best fits the physical network topology. Thus, the simulation tool provided the expected results coherently over the modeled bus and switch topologies. ClusterSim simplified time measurements because it didn't require any special techniques for measuring collective communications, so that they could be accurately measured.

Further experiments could also be possible if we considered: other communication patterns, different point-to-point primitives, other network topologies and technologies, buffer management, memory copies, packet routing, error rates etc. The simulation of some of these features would require the implementation of new modules for the simulator (e.g.: new network topologies). On the other hand some of the others can already be simulated without source-code changes.

Table 3. Response time ( $\mu \mathrm{s})$ of patterns on (a) reduction, (b) broadcast and (c) allgather

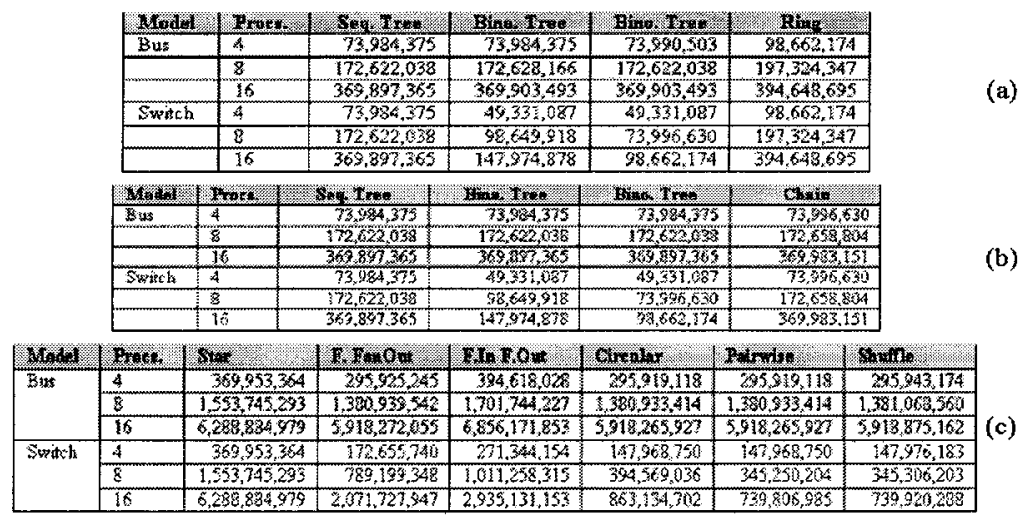

\subsection{Distributed Shared Memory Operations}

In order to simplify our experiments, we modeled only one consistency model and two coherence protocols with object replication in the nodes. The consistency model used in this simulation was sequential consistency, the strictest and most easily understandable consistency model. The modeled 
coherence protocols were: write-update and write-invalidate, which are often used and are easy to understand and implement.

In this case study, we created 4 workloads for testing over the bus based cluster. Each workload was a parallel job, containing different sequences of DSM operations for the shared object. Each one of the 4 workloads was a combination sequence of 4 DSM operations: LW (local write), RW (remote write), LR (local reads) and RR (remote reads). For each workload we varied the predominance level of remote and local operations between $20 \%$ and $80 \%$. For example, a workload LW80 is a workload composed of $20 \%$ of local writes on shared objects and $80 \%$ of remote operations on shared objects. Thus, our 4 workloads were: LW80, RW80, LR80 and RR80. The DSM operations were modeled to execute operations on objects with powers-of-two sizes (from 1 Byte to $256 \mathrm{~KB}$ ). We will only present the results for $4 \mathrm{~KB}$ objects, because the other sizes grew proportionally and their analysis would be repetitive. As we stated before in all simulations of DSM operations we use only the bus based cluster.

In order to simplify the visualization of the simulated results, in Fig.9, we used the letter $U$ to indicate workloads executed with Write-Update coherence protocol and the letter I to indicate workloads executed with Write-Invalidate coherence protocol.

In Fig. 8a, we present the number of control and object messages for all workloads using both simulated coherence protocols. In Fig. $8 \mathrm{~b}$ we present the job's response time for the simulated workloads. Considering the number of control messages sent (messages exchange between the node for controlling the remote operations), when we used the Write-Update protocol, $\mathrm{LR}$ and LW yielded the best results. In these workloads the remote shared objects were not frequently changed by the tasks, so the Shared Memory Manager didn't have to send many control messages to the nodes. For LR80, LW80 and RW80 workloads the write-invalidate presented the worst results (high number of control and object message), because on every change of a remote shared object its replicas were invalidated. So, whenever a node used an object after a subsequent write it had to obtain an object replica on that object. Only for the RR 80 workload the number of messages was the same for both coherence protocols. That happened because in this workload only $20 \%$ of the operations were write operations. Also the objects messages presented the same results for both coherence protocols. Considering the job response time (Fig. 8b) we can see that the response time for the workloads executed with the sequential consistency model and the write-update coherence protocols yielded the smallest times. In this coherence protocol the number of exchanged messages was smaller, so the time wasted with network was smaller and the job's response time was better. 
As expected, the results showed that the best results were obtained with the sequential consistency model using the write-update coherence protocol. Thus, the simulation tool provided the expected results coherently over the modeled protocols and model. Further experiments could also be possible if we considered: other coherence protocols, different consistency models, objects accessing protocols and other network topologies. The simulation of some of these features would require the implementation of new modules for the simulator (ex: new coherence protocols). That can be done by extending the existing coherence protocol classes. On the other hand some of the other coherence protocol classes can already be simulated without code changes.
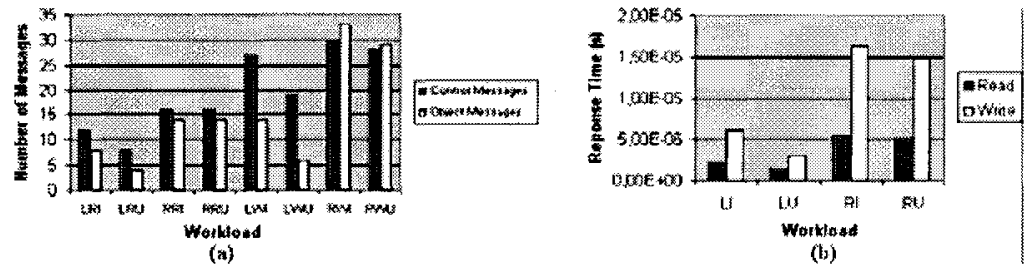

Figure 8. (a) Number of Messages x Workload (b) Response Time x Workload

\section{CONCLUSIONS}

In this paper we presented a new version of ClusterSim with the inclusion of Message-Passing (MP) and Distributed Shared Memory (DSM) modules. We verified the proposal and implementation of both modules by simulating: MPI collective communication functions using different communication patterns, DSM operations, consistency models and coherence protocols for object sharing. We also presented the new software architecture and simulation model. The main features of ClusterSim are: a hybrid workload model, a graphical environment, the modeling of heterogeneous clusters, and a statistical and performance module.

The graphical environment makes it easier to model, create and analyze the performance of clusters and workloads (parallel jobs and users). SRGSim and PP-MESS-SIM have detailed probabilistic workload simulation models that do not allow a detailed structural description of a job. Though the probabilistic description of a workload requires less simulation time and it is less representative than a structural description. On the other hand, the hybrid workload model of ClusterSim is composed of a probabilistic model and a structural description. It makes the simulation more deterministic than just probabilistic, thus making it feasible to represent real parallel jobs. Like Gridsim, ClusterSim supports heterogeneous nodes, but it also allows the configuration of interconnection networks with different parameters (ex: 
topology, latency etc), as well as SRGSim and Simgrid. Like ClusterSim, MPI-SIM implements MPI collective communication functions with basis on point-to-point primitives. Nevertheless, the latter requires real $\mathrm{C} / \mathrm{C}++/ \mathrm{UC}$ programs in order to run a simulation, whereas the former uses a hybrid language independent workload model. This model represents the abstract logic of the simulated program and is easier to understand. Besides, MPISIM only simulates point-to-point networks. Like DSMSim, ClusterSim also simulates distributed shared memory, but it provides more consistency models and coherence protocols, more statistical and performance information, and a graphical environment. Finally, ClusterSim has a statistical and performance module that provides data about the main metrics for the performance analysis of a cluster.

The main contributions of this paper are: the presentation, verification and analysis of MP and DSM modules in ClusterSim v.1.1. The new modules presented in this paper extended the functionalities of ClusterSim and enhanced its hybrid workload model. In the current version, it is feasible to model a workload by using instructions for: $\mathrm{CPU} /$ memory, $\mathrm{I} / \mathrm{O}$, point-topoint and collective communication, and DSM operations with memory coherence and consistency. MP and DSM were implemented on top of already validated modules and their verification was made by comparing analytical and experimental results. The verification led to a reliable simulation with very low error values. Thus, the simulator is yet a controllable and trustful environment with mechanisms for repeating and modifying parameters of real experiments.

The Java source code of ClusterSim is available and its classes are extensible. They allow the creation of new: network topologies, parallel job scheduling algorithms, collective communication functions and patterns, consistency models, coherence protocols, objects accessing protocols etc. As future works : implement a network topology editor and support to different routing algorithms, include new types of automatically generated workloads (ex: parallel programming models etc), include new memory consistency models and coherence protocols, simulate grid architectures, generate statistical and performance graphics, implement distributed simulation etc. More information at: http://ppgee.pucminas.br/lsdc projetos.htm.

\section{REFERENCES}

1. L. F. W. Góes, Proposal and Development of a Reconfigurable Parallel Job Scheduling Algorithm. M. Sc. Thesis. Belo Horizonte, Brazil, (2004) (in Portuguese).

2. L. E. S. Ramos, L. F. W. Góes and C. A. P. S. Martins, Teaching And Learning Parallel Processing Through Performance Analysis Using Prober, $32^{\text {nd }}$ ASEE/LEEE Frontiers in Education Conference, S2F13-18 (2002). 
3. R. Buyya, and M. Murshed, GridSim: A Toolkit for the Modeling and Simulation of Distributed Resource Management and Scheduling for Grid Computing, The Journal of Concurrency and Computation: Practice and Experience, 14 (13-15), 1175-1220, Wiley Press (2002).

4. A.M. Law and W.D.Kelton, Simulation Modeling and Analysis, McGraw-Hill (1991).

5. Y.H. Low et al., Survey of Languages and Runtime Libraries for Parallel Discrete-Event Simulation, IEEE Computer Simulation, 170-186, (1999).

6. R. MacNab and F.W. Howell, Using Java for Discrete Event Simulation, $12^{\text {th }}$ UK Performance Engineering Workshop, Edinburgh, 219-228, (1996).

7. H. Casanova, Simgrid: a Toolkit for the Simulation of Application Scheduling, $3^{\text {rd }}$ IEEE/ACM International Symposium on Cluster Computing and the Grid, Los Angeles, 430-437, (2001).

8. H. Bodhanwala, et al., A General Purpose Discrete Event Simulator, Symposium on Performance Analysis of Computer and Telecommunication Systems, USA, (2001).

9. S. Prakash and R.L. Bagrodia, MPI-SIM: Using Parallel Simulation to Evaluate MPI Programs, Winter Simulation Conference (WSC98), 467-474, (1998).

10. J. Rexford, W.-C Feng, J.W. Dolter and K.G. Shin, PP-MESS-SIM: A Flexible and Extensible Simulator for Evaluating Multicomputer Networks, IEEE Trans. Parallel Distributed Systems, 8(1): 25-40, (1997).

11. D. Thaker and V. Chaudhary, DSMSim: A Distributed Shared Memory Simulator for Clusters of Symmetric Multi-Processors, International Conference on Parallel and Distributed Processing Techniques and Applications, 1561-1567, (2003).

12. L. F. W. Góes and C.A.P.S. Martins, RJSSim: A Reconfigurable Job Scheduling Simulator for Parallel Processing Learning, $33^{\text {rd }}$ ASEE/IEEE Frontiers in Education Conference, Colorado, F3C3-8, (2003).

13. L. F. W. Góes, L.E.S. Ramos, C. A. P. S. Martins, ClusterSim: A Java-Based Parallel Discrete-Event Simulation Tool for Cluster Computing, IEEE Cluster 2004 Conference, (2004) (to appear).

14. Y. Zhang, H. Franke, E.J. Moreira and A. Sivasubramaniam, Improving Parallel Job Scheduling by Combining Gang Scheduling and Backfilling Techniques, IEEE International Parallel and Distributed Processing Symposium, 303-311, (2000).

15. B. B. Zhou, P. Mackerras, C. W. Johnson and D. Walsh, An Efficient Resource Allocation Scheme for Gang Scheduling, 1st IEEE Computer Society International Workshop on Cluster Computing, 187-194, (1999).

16. L. Breslau et al, Advances in Network Simulation, EEE Computer, 33 (5), 59-67, (2000).

17. A. Sulistio, C.S. Yeo, and R. Buyya, Visual Modeler for Grid Modeling and Simulation Toolkit, Technical Report, GRIDS Lab, Dept. of Computer Science and Software Engineering, The University of Melbourne, Australia, (2002).

18. A. Sulistio, C.S. Yeo and R. Buyya, A Taxonomy of Computer-based Simulations and its Mapping to Parallel and Distributed Systems Simulation Tools, International Journal of Software: Practice and Experience, 34(7), 653-673, Wiley Press, (2004).

19. A Collection of Modeling and Simulation Resources on the Internet, URL: www.idsia.ch/\%7Eandrea/sim/simindex.html

20. H.D. Schwetman, Using CSIM to Model Complex Systems, Winter Simulation Conference, (1988).

21. H-C. Hsiao and C-T. King, MICA: A Memory and Interconnect Simulation Environment for CacheBased Architectures, IEEE $33^{\text {rd }}$ Annual Simulation Symposium, 317-325, (2000).

22. W.B. Tan and P. Strazdins, The Analysis and Optimization of Collective Communications on a Beowulf Cluster, International Conference on Parallel and Distributed Systems, 659-666, (2002).

23. S.S. Vadhiyar, G.E. Fagg and J. Dongarra, Automatically Tuned Collective Communications, SuperComputing2000, 2000.

24. M. Dubois, C. Scheurich, and F. Briggs, "Synchronization, Coherence and Event Ordering in Multiprocessors", IEEE Computer, 21(2):9--21, (1988). 